\title{
582.
}

\section{NOTE ON THE THEORY OF PRECESSION AND NUTATION.}

[From the Monthly Notices of the Royal Astronomical Society, vol. xxxv. (1874-1875), pp. $340-343$.

WE have in the dynamical theory of Precession and Nutation (see Bessel's Fundamenta (1818), p. 126),

$$
\begin{aligned}
& C \frac{d p}{d t}+(B-A) q r=L S\left(x^{\prime} y-x y^{\prime}\right) d m^{\prime}\left(\frac{1}{\Delta^{3}}-\frac{1}{r^{3}}\right) \\
& A \frac{d q}{d t}+(C-B) r p=L S\left(y^{\prime} z-y z^{\prime}\right) d m^{\prime}\left(\frac{1}{\Delta^{3}}-\frac{1}{r^{3}}\right) \\
& B \frac{d r}{d t}+(A-C) p q=L S\left(z^{\prime} x-z x^{\prime}\right) d m^{\prime}\left(\frac{1}{\Delta^{3}}-\frac{1}{r^{3}}\right)
\end{aligned}
$$

where $L$ is the mass of the Sun or Moon, $x, y, z$ the coordinates of its centre referred to the centre of the Earth as origin,

the distance of its centre, and

$$
r=\sqrt{x^{2}+y^{2}+z^{2}}
$$

$$
\Delta=\sqrt{\left(x-x^{\prime}\right)^{2}+\left(y-y^{\prime}\right)^{2}+\left(z-z^{\prime}\right)^{2}},
$$

the distance of its centre from an element $d m^{\prime}$, coordinates $\left(x^{\prime}, y^{\prime}, z^{\prime}\right)$ of the Earth's mass, the sum or integral $S$ being extended to the whole mass of the Earth-I have written $d m^{\prime}, r$ for Bessel's $d m, r_{1}$-, we have

and thence

$$
\Delta^{2}=r^{2}-2\left(x x^{\prime}+y y^{\prime}+z z^{\prime}\right)+x^{\prime 2}+y^{\prime 2}+z^{\prime 2}
$$

$$
\frac{1}{\Delta^{3}}-\frac{1}{r^{3}}=\frac{3}{r^{5}}\left(x x^{\prime}+y y^{\prime}+z z^{\prime}\right)-\frac{3}{2} \frac{1}{r^{7}}\left\{\left(x^{2}+y^{2}+z^{2}\right)\left(x^{\prime 2}+y^{\prime 2}+z^{\prime 2}\right)-5\left(x x^{\prime}+y y^{\prime}+z z^{\prime}\right)^{2}\right\}+\text { etc. }
$$


The principal term is the first one,

$$
\frac{3}{r^{5}}\left(x x^{\prime}+y y^{\prime}+z z^{\prime}\right)
$$

but Bessel takes account also of the second term,

$$
-\frac{3}{2} \frac{1}{r^{7}}\left\{\left(x^{2}+y^{2}+z^{2}\right)\left(x^{\prime 2}+y^{\prime 2}+z^{\prime 2}\right)-5\left(x x^{\prime}+y y^{\prime}+z z^{\prime}\right)^{2}\right\},
$$

viz. considering the Earth as a solid of revolution (as to density as well as exterior form), he obtains in regard to it the following terms of $\sin \omega \frac{d \psi}{d t}$ and $\frac{d \omega}{d t}$ respectively;

$$
\begin{aligned}
& \frac{3 L}{4 r^{4}} \frac{1}{C n} \cdot 2(C-A) K\left(5 \sin ^{2} \delta-1\right) \cos \delta \sin \alpha, \\
- & \frac{3 L}{4 r^{4}} \frac{1}{C n} \cdot 2(C-A) K\left(5 \sin ^{2} \delta-1\right) \cos \delta \cos \alpha,
\end{aligned}
$$

where

$$
2(C-A) K=S\left(3 \mu-5 \mu^{3}\right) 2 \pi \rho R^{5} d R d \mu,
$$

$K$ being in fact a numerical quantity, relating to the Earth only, and the value of which is by pendulum observations ultimately found to be $=0 \cdot 13603$.

Writing, for shortness,

$$
\left(x^{2}+y^{2}+z^{2}\right)\left(x^{\prime 2}+y^{\prime 2}+z^{\prime 2}\right)-5\left(x x^{\prime}+y y^{\prime}+z z^{\prime}\right)^{2}=\Omega,
$$

then the foregoing terms of $\sin \omega \frac{d \psi}{d t}$ and $\frac{d \omega}{d t}$ depend, as regards their form, on the theorem that for any solid of revolution (about the axis of $z$ ) we have

$$
\begin{aligned}
& S\left(x^{\prime} y-x y^{\prime}\right) \Omega d m^{\prime}, \quad S\left(y^{\prime} z-y z^{\prime}\right) \Omega d m^{\prime}, \quad S\left(z^{\prime} x-z x^{\prime}\right) \Omega d m^{\prime} \\
& =0, \\
& \quad \frac{1}{2} y\left(x^{2}+y^{2}+z^{2}-5 z^{2}\right) S\left[3\left(x^{\prime 2}+y^{\prime 2}+z^{\prime 2}\right)-5 z^{\prime 2}\right] z^{\prime} d m^{\prime}, \\
& -\frac{1}{2} x\left(x^{2}+y^{2}+z^{2}-5 z^{2}\right) S\left[3\left(x^{\prime 2}+y^{\prime 2}+z^{\prime 2}\right)-5 z^{\prime 2}\right] z^{\prime} d m^{\prime},
\end{aligned}
$$

respectively: viz. writing $x^{\prime 2}+y^{\prime 2}+z^{\prime 2}=R^{2}$, and $z^{\prime}=R \mu$, also $x^{2}+y^{2}+z^{2}=r^{2}$ and $x=r \cos \delta \cos \alpha, y=r \cos \delta \sin \alpha, z=r \sin \delta$, the values would be

$$
\begin{aligned}
& 0, \\
& \frac{1}{2} r^{3} \cos \delta \sin \alpha\left(1-5 \sin ^{2} \delta\right) S\left(3-5 \mu^{2}\right) \mu R^{3} d m^{\prime}, \\
- & \frac{1}{2} r^{3} \cos \delta \sin \alpha\left(1-5 \sin ^{2} \delta\right) S\left(3-5 \mu^{2}\right) \mu R^{3} d m^{\prime},
\end{aligned}
$$

which are of the form in question.

The verification is easy: the solid being one of revolution about the axis of $z$, any integral such as $S x^{\prime} z^{\prime 2} d m^{\prime}$ or $S x^{\prime} y^{\prime} z^{\prime} d m^{\prime}$ which contains an odd power of $x^{\prime}$ or of $y^{\prime}$ is $=0$; while such integrals as $S x^{\prime 2} z^{\prime} d m^{\prime}, S y^{\prime 2} z^{\prime} d m^{\prime}$ are equal to each other, or, what is the same thing, each $=\frac{1}{2} S\left(x^{\prime 2}+y^{\prime 2}\right) z^{\prime} d m^{\prime}$. That we have $S\left(x^{\prime} y-x y^{\prime}\right) \Omega d m^{\prime}=0$ is 
at once seen to be true; considering the next integral $S\left(y^{\prime} z-y z^{\prime}\right) \Omega d m^{\prime}$, the terms of $\left(y^{\prime} z-z y^{\prime}\right) \Omega$ which lead to non-evanescent integrals are

$$
\begin{aligned}
& -y z^{\prime} \cdot\left(x^{2}+y^{2}+z^{2}\right)\left(x^{\prime 2}+y^{\prime 2}+z^{\prime 2}\right) \\
& -5 y^{\prime} z \cdot 2 y z y^{\prime} z^{\prime} \\
& +5 y z^{\prime} \cdot\left(x^{2} x^{\prime 2}+y^{2} y^{\prime 2}+z^{2} z^{\prime 2}\right)
\end{aligned}
$$

giving in the integral the several terms

$$
\begin{aligned}
& -y\left(x^{2}+y^{2}+z^{2}\right) S\left(x^{\prime 2}+y^{\prime 2}+z^{\prime 2}\right) z^{\prime} d m^{\prime} \\
& -10 y z^{2} \cdot \frac{1}{2} S\left(x^{\prime 2}+y^{\prime 2}+z^{\prime 2}-z^{\prime 2}\right) z^{\prime} d m^{\prime} \\
& +5 y\left(x^{2}+y^{2}+z^{2}-z^{2}\right) \cdot \frac{1}{2} S\left(x^{\prime 2}+y^{\prime 2}+z^{\prime 2}-z^{\prime 2}\right) z^{\prime} d m^{\prime} \\
& +y z^{2} S z^{\prime 3} d m^{\prime}
\end{aligned}
$$

viz. collecting, the value is

$$
\begin{aligned}
\left(-1+\frac{5}{2}\right. & =\frac{3}{2}\left(x^{2}+y^{2}+z^{2}\right) y S\left(x^{\prime 2}+y^{\prime 2}+z^{\prime 2}\right) z^{\prime} d m^{\prime} \\
\left(-\frac{5}{2}=\right. & )-\frac{5}{2}\left(x^{2}+y^{2}+z^{2}\right) y \Sigma z^{\prime 3} d m^{\prime} \\
\left(-\frac{5}{2}-5\right. & =)-\frac{15}{2} y z^{2} S\left(x^{\prime 2}+y^{\prime 2}+z^{\prime 2}\right) z^{\prime} d m^{\prime} \\
\left(+\frac{5}{2}+5+5\right. & =)+\frac{25}{2} y z^{2} S z^{\prime 3} d m^{\prime}
\end{aligned}
$$

which is

$$
=\frac{1}{2} y\left(x^{2}+y^{2}+z^{2}-5 z^{2}\right) S\left[3\left(x^{\prime 2}+y^{\prime 2}+z^{\prime 2}\right)-5 z^{\prime 2}\right] z^{\prime} d m^{\prime} ;
$$

and similarly the last term is

$$
=-\frac{1}{2} x\left(x^{2}+y^{2}+z^{2}-5 z^{2}\right) S\left[3\left(x^{\prime 2}+y^{\prime 2}+z^{\prime 2}\right)-5 z^{\prime 2}\right] z^{\prime} d m^{\prime}
$$

which completes the proof. 